\title{
TESTE DE DETERIORAÇÃO CONTROLADA PARA AVALIAÇÃO DO VIGOR DE SEMENTES DE FEIJÃ̃ ${ }^{1}$
}

\author{
CANDICE MELLO ROMERO SANTOS²; NILSON LEMOS DE MENEZES ${ }^{3}$; FRANCISCO AMARAL VILLELA ${ }^{4}$
}

\begin{abstract}
RESUMO - O teste de deterioração controlada tem sido utilizado freqüentemente para avaliar o vigor de diversas espécies, podendo ser uma alternativa para avaliação do vigor em sementes de feijão. No entanto, as condições para a realização deste teste ainda não estão completamente definidas. Devido a isto o presente trabalho teve por objetivo determinar as condições mais adequadas para a condução do teste de deterioração controlada em sementes de feijão comparando sua eficiência com o teste de envelhecimento acelerado. Utilizaram-se cinco lotes de sementes de feijão, cultivar Iapar 44, com diferentes qualidades fisiológicas. A qualidade inicial de cada lote de sementes foi avaliada pelos testes de germinação, primeira contagem, envelhecimento acelerado, condutividade elétrica e emergência em campo. Foram ajustadas as umidades iniciais das sementes para 15,20 e $25 \%$, e para cada umidade inicial, foram utilizadas quatro combinações de períodos de deterioração de 24 e 48 horas e temperaturas de deterioração de 40 e $45^{\circ} \mathrm{C}$. Os resultados permitiram concluir que o tratamento com sementes com umidade inicial de $20 \%$, por 48 horas sob temperatura de $45^{\circ} \mathrm{C}$, apresenta alta correlação com o teste de envelhecimento acelerado.
\end{abstract}

Termos para indexação: vigor, Phaseolus vulgaris, qualidade fisiológica.

\section{CONTROLLED DETERIORATION TEST TO EVALUATION OF VIGOUR IN BEAN SEEDS}

\begin{abstract}
The controlled deterioration test has frequently been used to evaluate the vigour of several species and could be an alternative for evaluation of the vigour in bean seeds. However, the conditions for the accomplishment of this test are not still completely defined. Due to this the objective of the present study was to determine the conditions more adapted for the conduction of the controlled deterioration test in bean seeds compare its efficiency with the accelerated ageing test. Five lots of bean seeds were used with different physiological qualities. The initial quality of each seed lot was evaluated by: germination test, first germination count, ageing accelerated test, electric conductivity and field emergence. The initial moisture of the seeds was established to be 15,20 and $25 \%$ and for each initial moisture content, there were four combinations of deterioration period of 24 and 48 hours and deterioration temperature of 40 and $45^{\circ} \mathrm{C}$. The results allowed the conclusion that seeds with $20 \%$ initial moisture, for 48 hours under temperature of $45^{\circ} \mathrm{C}$ presented high correlation with the accelerated ageing test.

Index terms: vigour, Phaseolus vulgaris, physiologic quality.
\end{abstract}

\section{INTRODUÇÃO}

\footnotetext{
${ }^{1}$ Aceito para publicação em 11/11/2003.

${ }^{2}$ Eng $^{\circ}$ Agr $^{\circ}$, Dr., Bolsista CNPq, Embrapa Recursos Genéticos e Biotecnologia; Quadra 203, Lote4, Bloco A, apt 903, Águas Claras, Brasília, DF, 71939-360; e-mail: candice_romero@hotmail.com

${ }^{3}$ Eng $^{\circ}$ Agr $^{\circ}$, Dr., Professor Adjunto do Depto. Fitotecnia da Universidade Federal de Santa Maria, 97105-900; e-mail: nlmenezes@smail.ufsm.br

${ }^{4}$ Eng. Agrícola, Dr., Professor Adjunto do Depto. de Fitotecnia da Universidade Federal de Pelotas; Caixa Postal 354, 96010-900; e-mail: bloisvillela@uol.com.br
}

No Brasil, o feijão comum representa o terceiro produto em área semeada e o quarto em valor de produção agrícola (CONAB, 2002). O esforço da pesquisa em obter melhores níveis de produtividade e garantir o abastecimento interno do produto é justificado pela importância social do feijão como alimento substituto de proteínas e rico em ferro e pelo consumo generalizado da população brasileira. 
A qualidade de um lote de sementes compreende uma série de atributos que determinam seu valor para a semeadura, sendo de natureza genética, física, fisiológica e sanitária (Popinigis, 1985). Destes, pode ser destacado o potencial fisiológico, diretamente responsável pelo desempenho das sementes no armazenamento e no campo. A resistência das sementes de alta qualidade a condições adversas de campo, conseqüente emergência de plantas e produção, tem grande importância na agricultura atual, tornando as tentativas para a resolução desses problemas em objetivos básicos da pesquisa sobre vigor de sementes. Esta vem procurando obter informações sobre o manejo de lotes durante o beneficiamento e armazenamento das sementes, de modo a possibilitar a manutenção de alto vigor pelo maior período possível.

O teste de germinação é utilizado em laboratórios para avaliar o potencial fisiológico das sementes, no entanto, é conduzido em condições favoráveis de temperatura, umidade e luminosidade, permitindo ao lote expressar o potencial máximo de produzir plântulas normais. Entretanto, esse teste pode ser pouco eficiente para indicar o desempenho no campo, onde as condições ambientais nem sempre são ideais (Marcos Filho, 1999). Portanto, a percentagem de emergência de plantas em campo, geralmente, é menor do que a percentagem de germinação obtida pelo teste de germinação.

A maior limitação do teste de germinação, segundo Hampton \& Tekrony (1995), é sua inabilidade para detectar diferenças de qualidade entre lotes com alta germinação. Por isso, têm sido desenvolvidos testes de vigor com o objetivo de identificar possíveis diferenças no potencial fisiológico de lotes que apresentam porcentagem de germinação semelhante, fornecendo informações complementares às obtidas no teste de germinação. Fergunson (1993) e Marcos Filho (1994) relataram que os testes de vigor descrevem informações adicionais sobre a qualidade fisiológica de sementes, como seu potencial de armazenamento e de produzir plântulas normais em condições adversas.

O teste de envelhecimento acelerado tem sido bastante estudado e recomendado para diferentes espécies e vem sendo incluído em programas de controle de qualidade de empresas produtoras de sementes. Foi desenvolvido por Delouche \& Baskin (1973), procurando estimar o potencial relativo de armazenamento de lotes de sementes de trevo e de festuca. Baseia-se no princípio de que lotes de alto vigor manterão sua viabilidade quando submetidos, durante curtos períodos de tempo, a condições severas de temperatura e umidade re- lativa do ar, enquanto que os de baixo vigor terão sua viabilidade reduzida, possibilitando a separação de lotes de sementes em diferentes níveis de vigor (Tekrony 1993; Rodo et al., 2000). O envelhecimento acelerado é eficiente para avaliar o vigor de lotes de sementes de feijoeiro e acompanhar a sua variação durante o período de armazenamento (Krzyzanowski et al., 1982).

Os testes de envelhecimento acelerado e de deterioração controlada têm como princípio a aceleração do processo de deterioração (Rossetto \& Marcos Filho,1995). Esse último, no entanto, incorpora melhor controle do grau de umidade da semente e da temperatura durante o período de envelhecimento. Lotes de sementes com percentuais de germinação semelhantes poderão apresentar diferentes níveis de deterioração (Krzyzanowski \& Vieira, 1999). Se existe diferença de vigor entre os lotes de sementes, o teste de deterioração controlada poderá estabelecer diferentes níveis de germinação após o tratamento, porque estes poderão estar em diferentes estádios de deterioração. Diferenças de vigor de sementes somente são importantes quando ocorrem entre lotes que apresentam alta germinação e, portanto, disponíveis para a comercialização (Krzyzanowski \& Vieira,1999). No teste de deterioração controlada mantém-se constante o grau de umidade das sementes durante o período de deterioração, enquanto no teste envelhecimento acelerado, o grau de umidade das sementes é variável (Hampton \& Tekrony,1995).

Em trabalho realizado com sementes de soja, o teste de envelhecimento acelerado comparado ao teste de deterioração controlada, apresentou-se mais drástico (Rossetto \& Marcos Filho, 1995; Santos et al., 2001).

$\mathrm{Na}$ condução dos testes que avaliam o vigor das sementes, muitos fatores afetam o comportamento das sementes, entre eles o grau de umidade das sementes e temperatura de incubação. No teste de envelhecimento acelerado, as sementes mais úmidas mostram maior sensibilidade às condições de temperatura e de umidade relativa do ar, por apresentarem atividade metabólica intensificada sob estas condições de ambiente. Portanto, os efeitos do envelhecimento acelerado são mais acentuados em sementes com graus de umidade mais elevados (Marcos Filho et al., 1987; Rossetto \& Marcos Filho,1995).

Existe uma relação entre o grau de umidade das sementes e a temperatura de condução do teste de deterioração controlada, pois as sementes devem ter o grau inicial de água elevado sob determinada temperatura. Porém, se a temperatura for maior, conforme Rossetto \& Marcos Filho (1995) pode-se utilizar sementes com menor grau inicial de água. 
Powell \& Matthews (1981) citam que um dos aspectos importantes no uso deste teste é a determinação do conteúdo de água das sementes, que pode variar conforme o lote.

A intensificação dos estudos sobre o teste de deterioração controlada constitui-se numa alternativa interessante, considerando que é um teste relativamente simples, não exige equipamentos sofisticados e não apresenta dificuldades consideráveis para sua padronização. Baseando-se nesta premissa, o presente trabalho teve por objetivo determinar as condições mais adequadas para a condução do teste de deterioração controlada em sementes de feijoeiro, comparando sua eficiência com o teste de envelhecimento.

\section{MATERIAL E MÉTODOS}

A pesquisa foi realizada no Laboratório de Análise de Sementes e Produção (LASP) do Departamento de Fitotecnia da Universidade Federal de Santa Maria-RS (UFSM), utilizando-se cinco lotes de sementes de feijão, cultivar IAPAR 44.

Para verificar a qualidade inicial de cada lote de sementes foram realizados os testes de germinação, primeira contagem de germinação, emergência em campo, envelhecimento acelerado e condutividade elétrica. $\mathrm{O}$ teste de germinação foi realizado utilizando-se oito subamostras de 50 sementes (quatro repetições de 100 sementes), conforme Brasil (1992). A primeira contagem de germinação foi realizada conjuntamente com o teste de germinação e avaliaram-se as plântulas normais no quarto dia após a instalação do teste. A emergência em campo foi conduzida com quatro repetições de 100 sementes e a contagem realizada no $20^{\circ}$ dia após a semeadura.

Para a realização do teste de envelhecimento acelerado, utilizaram-se 200 sementes por lote, com umidade inicial aproximada de $13 \%$. As sementes foram colocadas sobre uma bandeja de tela de alumínio, fixada no interior de uma caixa plástica, funcionando como compartimento individual (minicâmara). No interior dessa mini-câmara foram colocados $40 \mathrm{~mL}$ de água destilada e, em seguida, as caixas foram mantidas em incubadora, a temperatura de $42^{\circ} \mathrm{C}$, por um período de 72 horas, conforme recomendações de Marcos Filho (1999). Após esse período, as sementes foram submetidas ao teste de germinação e avaliadas segundo Brasil (1992).

Conduziu-se o teste de condutividade elétrica conforme Vieira (1994), utilizando-se quatro repetições de 50 sementes. Cada amostra foi pesada em balança com precisão de duas casas decimais e, a seguir, colocada em recipiente con- tendo $75 \mathrm{~mL}$ de água destilada e mantida em câmara à temperatura de $25^{\circ} \mathrm{C}$, durante 24 horas. Após esse período, efetuou-se a leitura da condutividade elétrica da solução de embebição com condutivímetro, marca DIGIMED CD 21. Os resultados obtidos foram divididos pelo peso das amostras, e a condutividade elétrica expressa em $\mu \mathrm{S} . \mathrm{cm}^{-1} \cdot \mathrm{g}^{-1}$.

Para a condução do teste de deterioração controlada utilizaram-se 200 sementes por lote. O umedecimento das sementes foi realizado colocando-as sobre papel de filtro embebido com água destilada em germinador à temperatura de $25^{\circ} \mathrm{C}$. Determinou-se o grau de umidade das sementes de 15 , 20 e $25 \%$ por intermédio de pesagens freqüentes das sementes, conforme Rossetto \& Marcos Filho (1995). Após alcançarem a umidade desejada, as sementes foram acondicionadas em sacos de papel-alumínio vedados e colocadas em câmara a $10^{\circ} \mathrm{C}$ por 12 horas, para uniformização do grau de umidade. A seguir, as sementes acondicionadas em embalagens de papel-alumínio devidamente vedadas e mantidas em banho-maria, conforme Krzyzanowski \& Vieira (1999). Fixou-se a umidade inicial das sementes $(15,20$ e $25 \%)$ e a partir disto os tratamentos constituíram-se na combinação de dois períodos de deterioração ( 24 e 48 horas) sob duas temperaturas $\left(40^{\circ}\right.$ e $\left.45^{\circ} \mathrm{C}\right)$. Após os períodos de deterioração, as sementes foram submetidas ao teste de germinação, e avaliadas conforme descrito por Brasil (1992).

O delineamento experimental utilizado foi o inteiramente casualizado no esquema fatorial. Para cada umidade inicial fixada, empregou-se o esquema bifatorial ( 2 períodos de deterioração x 2 temperaturas de deterioração). Os dados dos testes de germinação, primeira contagem, envelhecimento acelerado, deterioração controlada e emergência em campo foram transformados para $\operatorname{arcsen}(\% / 100)^{1 / 2}$.

As médias de tratamentos foram comparadas pelo teste Tukey, ao nível de 5\% de probabilidade de erro. Realizou-se a análise de correlação linear simples entre os resultados dos tratamentos de deterioração controlada nas sementes em relação aos resultados do teste de envelhecimento acelerado.

\section{RESULTADOS E DISCUSSÃO}

Os resultados obtidos nas avaliações da qualidade inicial dos lotes (Tabela 1) revelaram diferenças entre os lotes na qualidade fisiológica. Através do teste de germinação observou-se que os lotes 1, 2, 3 apresentaram porcentagens de germinação semelhantes entre si, sendo que o lote 1 diferiu dos lotes 4 e 5 e o lote 5 apresentou a menor geminação (70\%). O teste de primeira contagem de germinação estratificou os lo- 
TABELA 1. Caracterização da qualidade inicial de cinco lotes de sementes de feijoeiro. UFSM, Santa Maria-RS. 2002.

\begin{tabular}{|c|c|c|c|c|c|}
\hline Lotes & $\begin{array}{c}\text { Germinação } \\
(\%)\end{array}$ & $\begin{array}{c}\text { Primeira } \\
\text { contagem } \\
(\%)\end{array}$ & $\begin{array}{c}\text { Envelhecimento } \\
\text { acelerado } \\
(\%)\end{array}$ & $\begin{array}{c}\text { Emergência } \\
\text { em campo } \\
(\%)\end{array}$ & $\begin{array}{c}\text { Condutividade } \\
\text { elétrica } \\
\left(\mu \mathrm{S} . \mathrm{cm}^{-1} \cdot \mathrm{g}^{-1}\right)\end{array}$ \\
\hline 1 & $88 \mathrm{a}$ & $58 a^{*}$ & $48 \mathrm{a}$ & $54 a$ & $116 \mathrm{c}$ \\
\hline 2 & $86 a b$ & $56 a$ & $44 a$ & $52 \mathrm{a}$ & $118 \mathrm{~b}$ \\
\hline 3 & $86 a b$ & $52 \mathrm{~b}$ & $30 \mathrm{~b}$ & $48 \mathrm{a}$ & $125 \mathrm{ab}$ \\
\hline 4 & $84 \mathrm{~b}$ & $48 \quad \mathrm{c}$ & $12 \mathrm{c}$ & $50 a$ & $127 \mathrm{a}$ \\
\hline 5 & $70 \quad c$ & $42 \quad \mathrm{~d}$ & $8 c$ & $39 \mathrm{~b}$ & $131 \mathrm{a}$ \\
\hline Média & 82,8 & 51,2 & 28,4 & 49 & 124 \\
\hline $\mathrm{CV}(\%)$ & 1,52 & 2,30 & 24,42 & 2,10 & 2,30 \\
\hline
\end{tabular}

tes em quatro níveis de vigor: os lotes 1 e 2 de vigor superior, o lote 3 de vigor intermediário e os lotes 4 e 5 com vigor inferior. Examinando os resultados do teste de envelhecimento acelerado observou-se que os lotes 1 e 2 apresentaram maior vigor, o lote 3 apresentou vigor intermediário e os lotes 4 e 5 , menor vigor. No teste de emergência em campo, os lotes 1, 2, 3 e 4 não apresentaram diferenças entre si e foram superiores ao lote 5. Vale ressaltar que, o vigor inferior do lote 5 também foi expressado durante o teste de primeira contagem de germinação. Observou-se nos resultados da condutividade elétrica, que os lotes 1 e 2 apresentaram valores de condutividade menores, portanto maior reparo, integridade, organização do sistema de membranas celulares e, conseqüentemente, maior vigor, o lote 3 apresentou vigor intermediário e os lotes 4 e 5 tiveram maior lixiviação de exsudados para a solução de embebição, aumentando os valores da condutividade elétrica, demonstrando que estes lotes possuíam menor vigor.

Os dados referentes a umidade média inicial das sementes antes e após os testes de vigor estão apresentadas na Tabela 2 . Pode-se verificar que as sementes que foram submetidas ao teste de envelhecimento acelerado atingiram o teor de água mais elevado, ao final do teste, em relação aos dados obtidos no teste de deterioração controlada. Semelhantes resultados foram observados por Rossetto \& Marcos Filho (1995), os quais verificaram que em sementes de soja submetidas ao envelhecimento acelerado com diferentes graus de umidade, também apresentavam diferentes graus de umidade no final do teste. Entretanto, notou-se que o teor de água final no teste de deterioração controlada, manteve-se praticamente os mesmos do início do teste. Concordando, deste modo, com as afirmações de Krzyzanowski \& Vieira (1999) que citam

TABELA 2. Teor de umidade médio das sementes, antes e após a realização dos testes de deterioração controlada e envelhecimento acelerado. UFSM, Santa Maria, RS. 2002.

\begin{tabular}{|c|c|c|c|c|c|c|c|c|c|}
\hline \multirow{3}{*}{ Lotes } & \multirow{3}{*}{$\begin{array}{c}\text { Umidade inicial } \\
\text { dos lotes }\end{array}$} & \multicolumn{6}{|c|}{ Deterioração controlada } & \multirow{2}{*}{\multicolumn{2}{|c|}{$\begin{array}{c}\text { Envelhecimento } \\
\text { acelerado }\end{array}$}} \\
\hline & & \multicolumn{2}{|c|}{$15 \%$} & \multicolumn{2}{|c|}{$20 \%$} & \multicolumn{2}{|c|}{$25 \%$} & & \\
\hline & & UI & UF & UI & UF & UI & UF & $\mathbf{U I}$ & $\mathbf{U F}$ \\
\hline 1 & 12 & 14,9 & 14,9 & 20,2 & 20,2 & 24,9 & 25,0 & 13,0 & 26,8 \\
\hline 2 & 12 & 15,0 & 15,0 & 20,1 & 20,1 & 25,1 & 25,0 & 12,7 & 27,7 \\
\hline 3 & 14 & 15,1 & 15,1 & 19,9 & 19,9 & 25,0 & 25,0 & 13,5 & 31,2 \\
\hline 4 & 14 & 15,0 & 15,0 & 20,4 & 20,4 & 25,0 & 25,0 & 13,7 & 29,3 \\
\hline 5 & 14 & 15,0 & 15,0 & 20,2 & 20,2 & 24,9 & 24,9 & 13,5 & 30,0 \\
\hline
\end{tabular}

UI - Umidade das sementes antes da realização do teste.

UF - Umidade das sementes após a realização do teste. 
que o teste de deterioração controlada incorpora melhor o controle do grau de umidade da semente e da temperatura durante envelhecimento.

Os dados da porcentagem de germinação após os lotes de sementes serem submetidos aos tratamentos de deterioração controlada, com umidade inicial de $15 \%$, sob diferentes temperatura e período de exposição, estão apresentados na Tabela 3. A umidade das sementes utilizada neste tratamento (15\%), associada ao período de deterioração (24h), parece ter sido insuficiente para a separação dos lotes, pois esta ocorreu em apenas dois níveis de vigor, nas duas temperaturas testadas e contagens realizadas no quarto dia. Houve separação dos lotes em três níveis de vigor quando se utilizou o período de 48 horas e temperatura de $45^{\circ} \mathrm{C}$, com contagens realizadas no quarto dia e, também, durante o período de 24 horas de deterioração, sob temperatura de $40^{\circ} \mathrm{C}$ e avaliação no sétimo dia. $\mathrm{O}$ período de 48 horas e temperatura de $45^{\circ} \mathrm{C}$, com contagem realizada no sétimo dia, estratificou os lotes em quatro níveis de vigor.

As porcentagens de germinação do tratamento (48h$45^{\circ} \mathrm{C}$ ), em ambos períodos de avaliação, foram menores que os demais tratamentos devido ao excessivo período de deterioração na referida temperatura.

Os resultados de germinação de lotes de sementes de feijoeiro, com umidade inicial de $20 \%$, submetidas à deterioração controlada estão exibidos na Tabela 4 . O tratamento que utilizou 24 horas sob temperaturas de 40 e $45^{\circ} \mathrm{C}$ e avaliações realizadas no quarto dia, separou os lotes em três níveis de vigor. No entanto, quando se utilizou 48 horas de pe- ríodo de exposição, sob temperatura de $40^{\circ} \mathrm{C}$ e $45^{\circ} \mathrm{C}$, foi possível separar os lotes em quatro níveis de vigor, sendo que no tratamento a $40^{\circ} \mathrm{C}$ não houve separação entre os lotes 1 e 2, 2 e 3 , e no último tratamento $\left(45^{\circ} \mathrm{C}\right)$ não houve estratificação entre os lotes 3 e 4 . Quando as contagens de plântulas normais foram realizadas no sétimo dia, o tratamento $\left(24 \mathrm{~h}-40^{\circ} \mathrm{C}\right)$ separou os lotes em dois níveis de vigor, sendo os lotes $1,2,3$ e 4 superiores ao lote 5 . Já o tratamento $\left(24 \mathrm{~h}-45^{\circ} \mathrm{C}\right)$ promoveu a separação dos lotes em três níveis de vigor, sendo os lotes 1 e 2 mais vigorosos, os lotes 3 e 4 de vigor intermediário, enquanto que o lote 5 apresentou vigor inferior. No tratamento $\left(48 \mathrm{~h}-40^{\circ} \mathrm{C}\right)$ houve separação dos lotes em três níveis de vigor, não se observando diferenças entre os lotes 2,3 e 4. O tratamento de 48 horas sob temperatura de $45^{\circ} \mathrm{C}$, promoveu a separação dos lotes em três níveis de vigor, sendo que os lotes 1 e 2 de vigor superior não diferiram entre si, bem como os lotes 4 e 5 de vigor inferior, estes resultados (48h e $45^{\circ} \mathrm{C}$ ) foram semelhantes aos verificados durante a avaliação da qualidade inicial dos lotes, no teste de envelhecimento acelerado (Tabela 1).

De acordo com Hampton \& Tekrony (1995), lotes de sementes que mantiveram sua germinação após a deterioração são considerados lotes de alto vigor e aqueles que diminuíram a habilidade para germinar são considerados de baixo vigor.

Os dados referentes à germinação dos lotes de sementes de feijão, com umidade inicial de $25 \%$, submetidos a tratamentos de deterioração controlada estão apresentados na Tabela 5. Observou-se que quando se realizaram as avaliações

TABELA 3. Dados médios de germinação (\%) de cinco lotes de sementes de feijão, com umidade inicial de $15 \%$, submetidos ao teste de deterioração controlada empregando-se as combinações períodos de deterioração (24 e 48h) e temperaturas $\left(4^{\circ} \mathrm{e} 4^{\circ} \mathrm{C}\right)$. UFSM, Santa Maria - RS. 2002.

\begin{tabular}{|c|c|c|c|c|c|c|c|c|}
\hline \multirow{3}{*}{ Lotes } & \multicolumn{2}{|c|}{$24 h$} & \multicolumn{2}{|c|}{$48 \mathrm{~h}$} & \multicolumn{2}{|c|}{$24 \mathrm{~h}$} & \multicolumn{2}{|c|}{$48 \mathrm{~h}$} \\
\hline & $40^{\circ} \mathrm{C}$ & $45^{\circ} \mathrm{C}$ & $40^{\circ} \mathrm{C}$ & $45^{\circ} \mathrm{C}$ & $40^{\circ} \mathrm{C}$ & $45^{\circ} \mathrm{C}$ & $40^{\circ} \mathrm{C}$ & $45^{\circ} \mathrm{C}$ \\
\hline & \multicolumn{4}{|c|}{$4^{\circ}$ dia } & \multicolumn{4}{|c|}{$7^{\circ}$ dia } \\
\hline 1 & $70 a^{*} B$ & $64 \mathrm{aC}$ & $60 \mathrm{aC}$ & $44 \mathrm{aD}$ & $88 \mathrm{aA}$ & $84 \mathrm{aA}$ & $82 \mathrm{aA}$ & $74 \mathrm{aB}$ \\
\hline 2 & $68 \mathrm{aBC}$ & $64 \mathrm{aC}$ & $52 \mathrm{abD}$ & $44 \mathrm{aE}$ & $86 a b$ A & $86 a A$ & $84 \mathrm{aA}$ & $72 \mathrm{abB}$ \\
\hline 3 & $68 \mathrm{aB}$ & $62 \mathrm{aB}$ & $50 \mathrm{bC}$ & $42 a b D$ & $84 \mathrm{ab} \mathrm{A}$ & $82 \mathrm{aA}$ & $80 \mathrm{aA}$ & $68 \mathrm{bc} \mathrm{B}$ \\
\hline 4 & $60 \mathrm{~b} \mathrm{~B}$ & $60 \mathrm{ab} B$ & $48 \mathrm{~b} \mathrm{C}$ & 38 b D & $82 \mathrm{~b} \mathrm{~A}$ & $84 \mathrm{aA}$ & $80 \mathrm{aA}$ & 64 c B \\
\hline 5 & $54 \mathrm{bC}$ & $54 \mathrm{bC}$ & 48 b D & $34 \quad \mathrm{c} \mathrm{E}$ & $74 \quad \mathrm{c} \mathrm{A}$ & $68 \mathrm{~b} \mathrm{~B}$ & 68 b B & 58 \\
\hline Média & 64 & 60,8 & 51,6 & 40,4 & 82,8 & 80,8 & 78,8 & 67,2 \\
\hline $\mathrm{CV}(\%)$ & & 2,26 & & & & 1,59 & & \\
\hline
\end{tabular}

* Médias de tratamentos não seguidas por mesma letra, minúscula na vertical e maiúscula na horizontal, não diferem significativamente entre si, pelo teste Tukey ao nível de $5 \%$ de probabilidade de erro. 
TABELA 4. Dados médios de germinação (\%) de cinco lotes de sementes de feijão, com umidade inicial de $20 \%$, submetidos ao teste de deterioração controlada empregando-se as combinações, períodos de deterioração ( 24 e $48 \mathrm{~h})$ e temperaturas $\left(40^{\circ} \mathrm{e} 4^{\circ} \mathrm{C}\right)$. UFSM, Santa Maria, RS. 2002.

\begin{tabular}{|c|c|c|c|c|c|c|c|c|}
\hline \multirow{3}{*}{ Lotes } & \multicolumn{2}{|c|}{$24 \mathrm{~h}$} & \multicolumn{2}{|c|}{$48 \mathrm{~h}$} & \multicolumn{2}{|c|}{$24 \mathrm{~h}$} & \multicolumn{2}{|c|}{$48 \mathrm{~h}$} \\
\hline & $40^{\circ} \mathrm{C}$ & $45^{\circ} \mathrm{C}$ & $40^{\circ} \mathrm{C}$ & $45^{\circ} \mathrm{C}$ & $40^{\circ} \mathrm{C}$ & $45^{\circ} \mathrm{C}$ & $40^{\circ} \mathrm{C}$ & $45^{\circ} \mathrm{C}$ \\
\hline & \multicolumn{4}{|c|}{$4^{\circ}$ dia } & \multicolumn{4}{|c|}{$7^{\circ}$ dia } \\
\hline 1 & $72 \mathrm{aB}^{*}$ & $66 \mathrm{aC}$ & $60 \mathrm{aC}$ & $44 \mathrm{aD}$ & $82 \mathrm{aA}$ & $76 \mathrm{aB}$ & $70 \mathrm{aB}$ & $46 \mathrm{aD}$ \\
\hline 2 & $72 \mathrm{aB}$ & $56 \mathrm{bD}$ & $52 \mathrm{abD}$ & $26 \mathrm{bF}$ & $82 \mathrm{aA}$ & $74 \mathrm{aB}$ & $60 \mathrm{bC}$ & $40 \mathrm{abE}$ \\
\hline 3 & $58 \mathrm{~b} \mathrm{~B}$ & $54 \mathrm{bBC}$ & $44 \mathrm{bC}$ & $\mathrm{cE}$ & $80 \mathrm{aA}$ & $60 \mathrm{~b} \mathrm{~B}$ & $56 \mathrm{~b} \mathrm{~B}$ & $36 \mathrm{bD}$ \\
\hline 4 & $54 \mathrm{bc} \mathrm{BC}$ & $48 \mathrm{bcc}$ & $\mathrm{c} \mathrm{D}$ & $\mathrm{cE}$ & $80 \mathrm{aA}$ & $60 \mathrm{~b} \mathrm{~B}$ & 54 b BC & 30 \\
\hline 5 & 46 c B & 42 c B & $\mathrm{d} D$ & $\mathrm{dE}$ & $64 \mathrm{~b} \mathrm{~A}$ & $\mathrm{c} A \mathrm{AB}$ & $\mathrm{c} \mathrm{B}$ & 28 c C \\
\hline Média & 60,4 & 53,2 & 39,2 & 21,2 & 77,6 & 65,6 & 56,8 & 36 \\
\hline CV (\%) & & 3,98 & & & & 3,42 & & \\
\hline
\end{tabular}

* Médias de tratamentos não seguidas por mesma letra, minúscula na vertical e maiúscula na horizontal, não diferem significativamente entre si, pelo teste Tukey ao nível de 5\% de probabilidade de erro.

TABELA 5. Dados médios de germinação (\%) de cinco lotes de sementes de feijão, com umidade inicial de 25\%, submetidos ao teste de deterioração controlada empregando-se as combinações, períodos de deterioração ( 24 e 48h) e temperaturas $\left(40^{\circ} \mathrm{e} 45^{\circ} \mathrm{C}\right)$. UFSM, Santa Maria, RS. 2002.

\begin{tabular}{|c|c|c|c|c|c|c|c|c|}
\hline \multirow{3}{*}{ Lotes } & \multicolumn{2}{|c|}{$24 \mathrm{~h}$} & \multicolumn{2}{|c|}{$48 h$} & \multicolumn{2}{|c|}{$24 \mathrm{~h}$} & \multicolumn{2}{|c|}{$48 \mathrm{~h}$} \\
\hline & $40^{\circ} \mathrm{C}$ & $45^{\circ} \mathrm{C}$ & $40^{\circ} \mathrm{C}$ & $45^{\circ} \mathrm{C}$ & $40^{\circ} \mathrm{C}$ & $45^{\circ} \mathrm{C}$ & $40^{\circ} \mathrm{C}$ & $45^{\circ} \mathrm{C}$ \\
\hline & \multicolumn{4}{|c|}{$4^{\circ}$ dia } & \multicolumn{4}{|c|}{$7^{\circ} \mathrm{dia}$} \\
\hline 1 & $62 a^{*} B$ & $26 \mathrm{aE}$ & $10 \mathrm{aF}$ & $0 \mathrm{aG}$ & $82 \mathrm{aA}$ & $44 \mathrm{aD}$ & $52 \mathrm{aC}$ & $0 \mathrm{aG}$ \\
\hline 2 & $64 \mathrm{aB}$ & $16 \mathrm{abD}$ & $8 \mathrm{aE}$ & $0 \mathrm{aF}$ & $84 \mathrm{aA}$ & $46 \mathrm{aC}$ & $40 \mathrm{ab} C$ & $0 \mathrm{aF}$ \\
\hline 3 & $60 \mathrm{aA}$ & $12 \mathrm{bcD}$ & $12 \mathrm{aD}$ & $0 \mathrm{aE}$ & $78 \mathrm{aA}$ & $40 \mathrm{aB}$ & 32 b c C & $0 \mathrm{aE}$ \\
\hline 4 & $50 \mathrm{abB}$ & $8 \mathrm{bcD}$ & $8 \mathrm{aD}$ & $0 \mathrm{aE}$ & $80 \mathrm{aA}$ & $38 \mathrm{aC}$ & $32 \mathrm{bcC}$ & $0 \mathrm{aE}$ \\
\hline 5 & $40 \mathrm{~b} \mathrm{~B}$ & $6 \quad \mathrm{c} \mathrm{E}$ & $8 \mathrm{aE}$ & $0 \mathrm{aF}$ & $76 \mathrm{aA}$ & $32 \mathrm{aC}$ & $24 \quad c$ D & $0 \mathrm{aF}$ \\
\hline Média & 55,2 & 13,6 & 9,2 & 0 & 80 & 40 & 36 & 0 \\
\hline $\mathrm{CV}(\%)$ & & 14,25 & & & & 9,22 & & \\
\hline
\end{tabular}

* Médias de tratamentos não seguidas por mesma letra, minúscula na vertical e maiúscula na horizontal, não diferem significativamente entre si, pelo teste Tukey ao nível de 5\% de probabilidade de erro.

no quarto dia o tratamento de 24 horas de período de exposição sob temperatura de $40^{\circ} \mathrm{C}$, promoveu a separação dos lotes em dois níveis de vigor, quando se utilizou a temperatura de $45^{\circ} \mathrm{C}$ houve estratificação em três níveis de vigor; no entanto, os lotes 3, 4 e 5 não diferiram entre si. Na contagem realizada no sétimo dia, apenas o tratamento 48 horas sob temperatura de $40^{\circ} \mathrm{C}$ separou os lotes em três níveis de vigor, porém, este tratamento não mostrou diferenças entre os lotes 1 e 2, 2, 3 e 4, 3, 4 e 5. Os demais tratamentos não provocaram separação dos lotes conforme seu vigor. Durante o tratamento de 48 horas de período de exposição sob temperatura de $45^{\circ} \mathrm{C}$, com contagens realizadas no quarto e sétimo dias, a associação da temperatura de deterioração e do tempo de exposição foi excessiva, promovendo efeitos drásticos e a morte das sementes, concordando com Rossetto \& Marcos Filho (1995) os quais observaram que quanto maior o teor de água das sementes maiores são os efeitos deletérios da deterioração controlada.

Pode-se observar em todos os tratamentos que, conforme se aumentou à umidade inicial das sementes $(15,20 \mathrm{e}$ $25 \%$ ), ocorreu um decréscimo na porcentagem de germinação das sementes. Aquelas sementes com teor de água mais elevado apresentaram maior sensibilidade às condições ambientais desfavoráveis, promovendo estresse elevado nas 
sementes, chegando a provocar a morte das mesmas. Para Marcos Filho et al. (1987) sementes mais úmidas mostram mais sensibilidade as condições de alta temperatura e umidade relativa, pois apresentam atividade metabólica intensifica$\mathrm{da}$, sob as condições de envelhecimento, e as sementes com menor grau de umidade são menos prejudicadas.

Os resultados do teste de envelhecimento acelerado (Tabela 1) revelaram que este teste promove efeito mais drástico nas sementes, ou seja, a porcentagem final de germinação foi menor quando comparado ao teste de deterioração controlada, exceto quando se utilizou a umidade inicial de $25 \%$, associada a temperaturas de $48^{\circ} \mathrm{C}$, para tal teste. Provavelmente este efeito mais severo tenha sido ocasionado pelos altos graus de umidade atingidos pelas sementes ao final do teste de envelhecimento acelerado, os quais aceleraram a atividade metabólica das sementes.

Os tratamentos que utilizaram $20 \%$ de umidade inicial das sementes pelos períodos de 48 horas de deterioração sob temperatura de $45^{\circ} \mathrm{C}$ com avaliação no sétimo dia e 24 horas de deterioração sob temperaturas de 40 e $45^{\circ} \mathrm{C}$ com avaliações no quarto dia, apresentaram os maiores coeficientes de correlação simples $r=0,988, r=0,878$ e $r=0,890$, respectivamente (Tabela 6) quando comparado aos resultados do teste de envelhecimento acelerado.

Os resultados observados no presente trabalho relacionam-se com os observados por Santos et al. (2001) e discordam de Powell \& Matthews (1981), os quais relataram que o teste de deterioração controlada não revela claramente as diferenças entre lotes de vigor médio, mas apenas entre lotes de vigor baixo e alto.

O teste de deterioração controlada é recomendado, principalmente, para hortaliças (Powell \& Matthews, 1981; Mendonça et al., 2000), no entanto pesquisadores brasileiros como Rossetto \& Marcos Filho (1995) e Marcos Filho et al. (2001) têm estudado este teste para sementes de soja. Através dos resultados foi possível observar que o teste de deterioração controlada pode ser uma nova alternativa para a avaliação do vigor em sementes de feijoeiro, por promover a separação dos lotes e ser de fácil padronização e interpretação.
TABELA 6. Coeficientes de correlação (r) simples e significativo entre os tratamentos de deterioração controlada e o teste de envelhecimento acelerado.UFSM. Santa Maria, RS, 2002.

\begin{tabular}{|c|c|}
\hline Tratamentos & Envelhecimento acelerado \\
\hline \multicolumn{2}{|c|}{....................... Contagem no $4^{\mathrm{o}}$ dia } \\
\hline DC $15 \% / 24 \mathrm{~h}-40^{\circ} \mathrm{C}$ & - \\
\hline DC $15 \% / 24 h-45^{\circ} \mathrm{C}$ & - \\
\hline DC $15 \% / 48 \mathrm{~h}-40^{\circ} \mathrm{C}$ & - \\
\hline DC $15 \% / 48 h-45^{\circ} \mathrm{C}$ & 0,690 \\
\hline \multicolumn{2}{|c|}{ Contagem no $7^{\circ}$ dia } \\
\hline DC $15 \% / 24 h-40^{\circ} \mathrm{C}$ & 0,723 \\
\hline DC $15 \% / 24 h-45^{\circ} \mathrm{C}$ & - \\
\hline DC $15 \% / 48 \mathrm{~h}-40^{\circ} \mathrm{C}$ & - \\
\hline DC $15 \% / 48 \mathrm{~h}-45^{\circ} \mathrm{C}$ & 0,533 \\
\hline \multicolumn{2}{|c|}{ Contagem no $4^{\mathrm{o}}$ dia } \\
\hline DC $20 \% / 24 \mathrm{~h}-40^{\circ} \mathrm{C}$ & 0,878 \\
\hline DC $20 \% / 24 \mathrm{~h}-45^{\circ} \mathrm{C}$ & 0,890 \\
\hline DC $20 \% / 48 \mathrm{~h}-40^{\circ} \mathrm{C}$ & 0,574 \\
\hline DC $20 \% / 48 \mathrm{~h}-45^{\circ} \mathrm{C}$ & 0,421 \\
\hline \multicolumn{2}{|c|}{ Contagem no $7^{\circ}$ dia } \\
\hline DC $20 \% / 24 \mathrm{~h}-40^{\circ} \mathrm{C}$ & - \\
\hline DC $20 \% / 24 \mathrm{~h}-45^{\circ} \mathrm{C}$ & 0,712 \\
\hline DC $20 \% / 48 \mathrm{~h}-40^{\circ} \mathrm{C}$ & 0,754 \\
\hline DC $20 \% / 48 \mathrm{~h}-45^{\circ} \mathrm{C}$ & 0,988 \\
\hline \multicolumn{2}{|c|}{ Contagem no $4^{\mathrm{o}}$ dia } \\
\hline DC $25 \% / 24 \mathrm{~h}-40^{\circ} \mathrm{C}$ & - \\
\hline $\mathrm{DC} 25 \% / 24 \mathrm{~h}-45^{\circ} \mathrm{C}$ & 0,657 \\
\hline DC $25 \% / 48 \mathrm{~h}-40^{\circ} \mathrm{C}$ & - \\
\hline DC $25 \% / 48 h-45^{\circ} \mathrm{C}$ & - \\
\hline \multicolumn{2}{|c|}{ Contagem no $7^{\circ}$ dia } \\
\hline $\mathrm{DC} 25 \% / 24 \mathrm{~h}-40^{\circ} \mathrm{C}$ & - \\
\hline $\mathrm{DC} 25 \% / 24 \mathrm{~h}-45^{\circ} \mathrm{C}$ & - \\
\hline $\mathrm{DC} 25 \% / 48 \mathrm{~h}-40^{\circ} \mathrm{C}$ & 0,649 \\
\hline DC $25 \% / 48 h-45^{\circ} \mathrm{C}$ & - \\
\hline
\end{tabular}

(-) não houve efeito significativo e positivo ao nível de 5\% de probabilidade de erro. 


\section{CONCLUSÃO}

O teste de deterioração controlada conduzido com grau de umidade inicial das sementes de $20 \%$, período de exposição de 48 horas à temperatura de $45^{\circ} \mathrm{C}$, permite a separação de sementes de feijoeiro com diferentes níveis de vigor e apresenta alta correlação com o teste de envelhecimento acelerado.

\section{REFERÊNCIAS}

BRASIL, Ministério da Agricultura e Reforma Agrária. Regras para análise de sementes. Brasília: SNAD/DNPV/CLAV,1992. 365p.

COMPANHIA NACIONAL DE ABASTECIMENTO. CONAB2002. http:/ www.conab.gov.br.

DELOUCHE, J.C.; BASKIN, C.C. Accelerated aging technique for predicting the relative storability of seed lots. Seed Science \& Technology, Zurich, v.1, p.427-452, 1973.

FERGUSON, J.M. AOSA Perspective of seed vigor testing. Journal of Seed Technology, Lansing, v.17, n.2, p.101-104, 1993.

TEKRONY, D.M. Accelerated aging test. Journal of Seed Technology, Lansing v.17, n.2, p.110-120, 1993.

HAMPTON, J.G.; TEKRONY, D.M. Controlled deterioration test. In: HAMPTON AND TEKRONY (ed). Handbook of vigour test methods. Zurich: ISTA.1995. p.70-78.

MARCOS FILHO, J.; CICERO, S.M.; SILVA, W.R. Avaliação da qualidade das sementes. Piracicaba: FEALQ,1987. 230p.

MARCOS FILHO, J. Teste de envelhecimento acelerado. In: VIEIRA, R.D. \& CARVALHO, N.M. Testes de vigor em sementes. Jaboticabal: FUNEP, 1994. 164p.

MARCOS FILHO, J. Teste de envelhecimento acelerado. In: KRZYZANOWSKI, F.C.; VIEIRA, R.D.; FRANÇANETO, J.B. Vigor de sementes: conceitos e testes. Londrina: ABRATES, 1999. p.1-24.
MARCOS FILHO, J.; NOVEMBRE, A.D.C.; CHAMA, M.C.P. Testes de envelhecimento acelerado e de deterioração controlada para a avaliação do vigor de sementes de soja. Scientia Agrícola, Piracicaba, v.58, n.2, p.421-426, 2001.

MENDONÇA, E.A.F.; RAMOS, N.P.; FESSEL, S.A.; SADER, R. Teste de deterioração controlada em sementes de Brocoli (Brassica oleraceae L.) var. itálica. Revista Brasileira de Sementes, Brasília, v.22, n.1, p.280-287, 2000.

KRZYZANOWSKI, F.C.H.; COSTA, J.D.; SCOTTI, C.A.; DA SILVEIRA, J.F. O envelhecimento precoce na avaliação de lotes de sementes de feijoeiro. Revista Brasileira de Sementes, Brasília, v.4, n.1, p.45-58,1982.

KRZYZANOWSKI, F.C.H.; VIEIRA, R.D. Deterioração controlada. In: KRZYZANOWSKI, F.C.H.; VIEIRA, R.D.; FRANÇA NETO, J.B. Vigor de sementes: conceitos e testes. Londrina: ABRATES, 1999. 218p.

POPINIGIS, F. Fisiologia da semente, Brasília, ABEAS, 1985. 289p.

POWELL, A.A.; MATTHEWS, S. Evaluation of controlled deterioration: a new vigour test for small seeded vegetables. Seed Science \& Technology, Zurich, v.9, p.633-640, 1981.

RODO, A.B.; PANOBIANCO, M.; MARCOS FILHO, J. Metodologia alternativa do teste de envelhecimento acelerado para sementes de cenoura. Scientia Agrícola, Piracicaba, v.57, n.2, p.289292, 2000.

ROSSETTO, C.A.V.; MARCOS FILHO, J. Comparação entre os métodos de envelhecimento acelerado e de deterioração controlada para avaliação da qualidade fisiológica de sementes de soja. Scientia Agrícola, Piracicaba, v.52, n.1, p.123-131,1995.

SANTOS, C.M.R.; MENEZES, N.L.; SANTOS, O.S. Envelhecimento artificial para determinação do vigor em sementes de feijoeiro. Informe Técnico (CCR) - Universidade Federal de Santa Maria (UFSM), Santa Maria, n.1, 2001, 7p.

VIEIRA, R.D. Teste de condutividade elétrica. In: VIEIRA, R.D. \& CARVALHO, N.M. Testes de vigor em sementes. Jaboticabal: FUNEP, 1994. 164p. 